Cite this: Nanoscale, 2014, 6, 6590

\title{
Highly graphitized nitrogen-doped porous carbon nanopolyhedra derived from ZIF-8 nanocrystals as efficient electrocatalysts for oxygen reduction reactions $\uparrow$
}

\begin{abstract}
Linjie Zhang, ${ }^{\text {ab }}$ Zixue Su, ${ }^{\mathrm{c}}$ Feilong Jiang, ${ }^{a}$ Lingling Yang, ${ }^{\mathrm{a}}$ Jinjie Qian, ${ }^{\text {ab }}$ Youfu Zhou, ${ }^{a}$ Wenmu Li ${ }^{\mathrm{a}}$ and Maochun Hong*a

Nitrogen-doped graphitic porous carbons (NGPCs) have been synthesized by using a zeolite-type nanoscale metal-organic framework (NMOF) as a self-sacrificing template, which simultaneously acts as both the carbon and nitrogen sources in a facile carbonization process. The NGPCs not only retain the nanopolyhedral morphology of the parent NMOF, but also possess rich nitrogen, high surface area and hierarchical porosity with well-conducting networks. The promising potential of NGPCs as metal-free electrocatalysts for oxygen reduction reactions (ORR) in fuel cells is demonstrated. Compared with commercial Pt/C, the optimized NGPC-1000-10 (carbonized at $1000{ }^{\circ} \mathrm{C}$ for $10 \mathrm{~h}$ ) catalyst exhibits comparable electrocatalytic activity via an efficient four-electron-dominant ORR process coupled with superior methanol tolerance as well as cycling stability in alkaline media. Furthermore, the controlled experiments reveal that the optimum activity of NGPC-1000-10 can be attributed to the synergetic contributions of the abundant active sites with high graphitic-N portion, high surface area and porosity, and the high degree of graphitization. Our findings suggest that solely MOF-derived heteroatom-doped carbon materials can be a promising alternative for Pt-based catalysts in fuel cells.
\end{abstract}

Received 19th January 2014

Accepted 5th March 2014

DOI: $10.1039 / c 4 n r 00348 a$

www.rsc.org/nanoscale from multiple competitive disadvantages, including their susceptibility to time-dependent potential drift, fuel crossover effect and CO deactivation, ${ }^{3}$ together with the high cost and scarcity of the noble Pt metal, which are the major stumbling blocks for commercializing PEMFCs. On this basis, intensive research efforts have been directly committed to the search for alternative catalysts to rival the Pt-based catalyst in activity and durability with reduced Pt usage, or non-precious metals, or even metal-free species over the past decades. ${ }^{4-6}$ Compared to Pt-based catalysts, metal-free catalysts have several notable advantages, as they do not suffer from fuel crossover effect or CO poisoning, have long-term operational stability, and are relatively cost-effective. ${ }^{7}$ In recent years, breakthroughs in the striking ORR electrocatalytic performance have been achieved for a large variety of heteroatom (N, B, P, S or F) single/dualdoped carbon materials, including carbon nanotubes $(\mathrm{CNTs}),{ }^{8-10}$ graphites, ${ }^{11}$ graphenes, ${ }^{12,13}$ graphene oxides, ${ }^{14,15}$ graphitic or amorphous carbons ${ }^{16,17}$ and also their composites. ${ }^{18,19}$ Both theoretic calculations and detailed experiments have demonstrated that the intentional incorporation of heteroatoms into carbons could effectively tune their intrinsic properties, including electronic characteristics, surface and local chemical features, which might reduce the energy barriers of oxygen adsorption and activation, and thus improve the catalytic activity towards ORR. Among the various Pt 
replacement candidates, nitrogen-doped carbons (NDCs) with excellent ORR performance have long been a focal point. Although the nature of the active ORR catalytic sites in NDCs is still debated, ${ }^{20}$ there is no doubt that the ORR performance of NDCs strongly depends on the type of nitrogen precursors used, the nitrogen content, the heat treatment temperature and the synthesis conditions. Obviously, tailoring the electronic structure of carbon materials by doping could be a practical strategy for producing advanced materials promising for ORR in fuel cells. Nevertheless, NDCs have conventionally been synthesized via chemical vapor deposition (CVD), arc-discharge/vaporization approach or plasma treatment under an $\mathrm{NH}_{3}$ atmosphere or in the presence of other nitrogen sources such as pyridine and acetonitrile. ${ }^{21}$ These methods usually involve multistep procedures and rigorous reaction conditions, which not only require high energy consumption but also are difficult to handle due to the use of small and toxic molecules $\left(\mathrm{N}_{2} \mathrm{H}_{4}\right.$, $\mathrm{HCN}, \mathrm{NH}_{3}$, etc.) as precursors. More importantly, they have a poor control over the $\mathrm{N}$ dosage amount, chemical homogeneity, dopant state and reproducibility, and can sometimes deteriorate the carbon structure. Another challenge for metal-free ORR catalysts is their relatively low turnover frequency in comparison with $\mathrm{Pt}^{22}$ To compensate low activity without using excess catalyst, it is desirable to produce catalytic sites with high surface density, that are evenly distributed and accessible to gas diffusion through a porous framework. Consequently, the development of highly active metal-free catalysts with similar properties based on facile, versatile, inexpensive, and reproducible synthesis is of high importance.

Recently, metal-organic frameworks (MOFs), ${ }^{23}$ resulting from periodically arranged organometallic complexes held together by metal ions in three-dimensional (3D) space, have emerged as a new family of solid matrices to be used as hard templates for casting of porous carbons owing to their high specific surface area (SSA) and porosity, chemical tunability, and well-defined pore structure, which is suitable for accommodating small organic molecules (i.e., the carbon precursors), such as furfuryl alcohol, glycerol and ethylenediamine. Ever since the pioneering work by Xu's group ${ }^{24}$ a renewed interest in porous carbons was triggered due to the large number of available MOFs with versatile pore structures. And the nanoporous carbons thus obtained showed attractive features, including ultra-high SSA and pore volumes, and exhibited a wide spectrum of energy-related applications in hydrogen storage, ${ }^{25-27}$ supercapacitors, ${ }^{24,28,29}$ lithium-sulfur batteries, ${ }^{30,31}$ and fuel cells, ${ }^{32}$ etc. Moreover, in view of the rich carbon content of the organic building blocks used, a MOF itself could also capable as an alternative precursor to construct nanoporous carbons without the need of a second carbon source or a poreforming agent. So far, several MOFs, such as MOF-5, ${ }^{33} \mathrm{Al}-\mathrm{PCP}^{34}$ and ZIF-8 (ref. 35) have been demonstrated as promising selfsacrificial templates to afford highly nanoporous carbon materials. More importantly, considering that the organic ligands also contain various other types of atoms (N, O, P or S, etc.) other than carbon, therefore, MOFs, which are rich in carbon and nitrogen, easily prepared and very cost-effective, could be ideal precursors with potential to produce nitrogen-doped porous carbon materials with uniformly distributed catalytic centers and high active site density. However, although the early attempts in using solely MOFs (such as MOF-5) as carbon precursors were successfully made, control over porous structures, particle morphologies and sizes as well as the heteroatom doping states were rarely presented. Moreover, previously reported ORR catalysts based on MOF-derived carbons usually contain metal species (e.g., Fe or Co), ${ }^{36,37}$ the presence of which not only compromises their electroactivity and shelf life due to the metal leaching over time in the often used alkaline media for ORR, but also makes the role of MOF-derived carbon in ORR more difficult to understand. As far as we know, solely MOFderived metal-free heteroatom-doped carbon materials towards ORR have not yet been reported. The omission of this study prompts us to carry out a systematic investigation on how the MOF-derived metal-free carbons can perform for ORR.

It is thus the philosophy of the present contribution to architect a metal-free carbon material with controllable particle morphology and size, high surface area, stable porous structure, good conductivity and rich nitrogen doping by an economical and low-hazardous MOF-driven approach for the ORR in alkaline media. With this aim, we judiciously chose a chemically and thermally robust as well as highly porous zeolite-type MOF $^{38}$ (ZIF$8 ; \mathrm{Zn}(\mathrm{MeIM})_{2} ; \mathrm{MeIM}=2$-methylimidazole) nanocrystal (NC) as both the precursor and template, in order to take advantage of both inherent and nanoscale properties of the nanoscale MOF (NMOF) in the templated synthesis of N-doped porous carbons. The use of such a polymer as a precursor promises an increase of the high active site density and a more uniform distribution of nitrogen sites on the surface. Different from the amino ligands used for many other MOF analogues, the aromatic methylimidazole ligand in ZIF-8 is free of oxygen and highly enriched in nitrogen (molar ratio: $\mathrm{N} / \mathrm{C}=1 / 2$ ) that is directly incorporated into the aromatic ring, thus can facilitate the incorporation of abundant nitrogen-containing active sites into the carbon matrix (for precursors containing nitrogen in aromatic structures, the elimination of nitrogen is more difficult than that for precursors containing nitrogen attached to the aromatic rings at high temperatures). ${ }^{39}$ Furthermore, the use of MeIM as a carbon precursor also promotes a much higher degree of graphitization in the resultant carbon skeletons compared to non-aromatic carbon precursors (e.g., sucrose and glucose). ${ }^{40}$ As a contribution to the domain of nitrogen-doped graphitic porous carbons (NGPCs) and their utilization as highly efficient metal-free cathode ORR electrocatalysts in PEMFCs, the present work will report and discuss in detail the synthesis, structural characterization and the ORR performance of the resultant highly graphitized and in situ nitrogen-doped carbon nanopolyhedra (NP) with high surface density and uniform distribution of active sites. An effort is also made to elucidate the correlation between the mesoscopic structures and the electrochemical activity of NGPCs.

\section{Experimental section}

\section{Chemicals}

The following materials were used as received without further purification. 2-Methylimidazole and terephthalic acid were 
purchased from TCI. JM $20 \mathrm{wt} \% \mathrm{Pt} / \mathrm{C}$ and $5 \mathrm{wt} \%$ Nafion ionomer were purchased from Alfa-Aesar and Aldrich, respectively. $\mathrm{Zn}\left(\mathrm{NO}_{3}\right)_{2} \cdot 6 \mathrm{H}_{2} \mathrm{O}, \mathrm{KOH}$, concentrated $\mathrm{H}_{2} \mathrm{SO}_{4}$ solution (98\%), $N, N$ dimethylformamide (DMF), methanol, ethanol, isopropanol and other reagents were of analytical grade. High-purity nitrogen, oxygen gases and distilled water were used in all experiments.

\section{Synthesis of nanoporous carbon nanopolyhedra}

The preparation of ZIF-8 precursor was analogous to that reported in the literature with slight modifications of the ratio of the reactants used and the reaction time..$^{41}$ Briefly, a known quantity $(0.735 \mathrm{~g})$ of $\mathrm{Zn}\left(\mathrm{NO}_{3}\right)_{2} \cdot 6 \mathrm{H}_{2} \mathrm{O}$ was employed to obtain a mixture with $\mathrm{Zn} / \mathrm{MeIM} / \mathrm{methanol}$ in a molar ratio of approximately $1: 8: 700$. The mixture was left to stand for $1 \mathrm{~h}$ at room temperature, and the solid was recovered from the milky colloidal solution by centrifugation and washed with fresh methanol. Then the products were dried under reduced pressure. For the synthesis of NGPCs, ZIF-8 NCs $(0.2 \mathrm{~g})$ were weighed in a ceramic boat and transferred into a quartz tube. The tube was sealed airtight and placed inside a furnace, where the carbonization was performed at a designated temperature with a heating rate of $5{ }^{\circ} \mathrm{C} \mathrm{min}{ }^{-1}$ under a steam of nitrogen $\left(45 \mathrm{~L} \mathrm{~h}^{-1}\right)$ after excluding air by flowing $\mathrm{N}_{2}$ for $30 \mathrm{~min}$. The furnace was allowed to cool to room temperature, after which the products were harvested as black powders with yields of $c a$. $36-80 \%$. Finally, the resultant materials were washed thoroughly in $3 \mathrm{M}$ $\mathrm{H}_{2} \mathrm{SO}_{4}$ solution to remove residual $\mathrm{Zn}$ species, if any. These asprepared samples are denoted as NGPC- $T_{\mathrm{c}}-t$, where $T_{\mathrm{c}}$ represents the carbonization temperature $\left(700,800,900,1000{ }^{\circ} \mathrm{C}\right)$ and $t$ represents the carbonization time $(1,5,10 \mathrm{~h})$.

A nitrogen- and metal-free carbon catalyst, derived from the carbonization of a well-known zinc-containing $\mathrm{MOF},{ }^{42}$ $\left[\mathrm{Zn}_{4} \mathrm{O}(\mathrm{bdc})_{3}\right]$ (MOF-5, bdc $=1,4$-benzenedicarboxylate) using the aforementioned procedure, was also used for ORR study.

All the resultant carbon samples were dried and activated at $200{ }^{\circ} \mathrm{C}$ for $10 \mathrm{~h}$ under vacuum before being used for further electrochemical study.

\section{Physical characterizations}

The products were characterized by using scanning electron microscopy (SEM) on a JEOL JSM 6700F microscope, transmission electron microscopy (TEM) on an FEI Tecnai F20 electron microscope, powder X-ray diffraction (PXRD) on a Rigaku DMAX2500 X-ray diffractometer using a copper target $(\lambda=$ $0.154 \mathrm{~nm}$ ), X-ray photoelectron spectroscopy (XPS) on a Thermo Scientific ESCALAB 250, thermal gravimetric analysis (TGA) on a Netzsch STA449C instrument using pure nitrogen as a carrier gas with a heating rate of $5{ }^{\circ} \mathrm{C} \mathrm{min}^{-1}$, Fourier-transform infrared spectroscopy (FT-IR) on a PerkinElmer Spectrum One instrument with samples prepared as $\mathrm{KBr}$ pellets at wavenumbers ranging from 4000 to $400 \mathrm{~cm}^{-1}$, and inductively coupled plasma optical emission spectroscopy (ICP-OES) on a Jobin Yvon Ultima2. Raman spectra were recorded on a Renishaw UV-1000 Photon Design spectrometer using an argon ion laser with an excitation wavelength of $532 \mathrm{~nm}$. Pore characteristics of the materials were assessed from nitrogen sorption isotherms measured at $77 \mathrm{~K}$ on a Micromeritics ASAP2020.

\section{Electrochemical analysis}

Electrochemical properties were investigated by cyclic voltammetry (CV) and linear-sweep voltammetry (LSV) on a Pine instrument in a conventional three-compartment electrochemical Teflon cell, using a rotating (ring) disk electrode $(\mathrm{R}(\mathrm{R})$ $\mathrm{DE}$ ) setup with $\mathrm{Ag} / \mathrm{AgCl}$ as the reference electrode and Pt foil as the counter electrode. Before the preparation of the working electrode (WE), the glassy carbon electrode (GCE; $5 \mathrm{~mm}$ diameter, $0.196 \mathrm{~cm}^{2}$ geometric surface area) was polished on 1.0, 0.3, and $0.05 \mu \mathrm{m}$ alumina powder, respectively, and was sonicated several times alternately in de-ionized water and EtOH ( 2 min in each) to a mirror finish and dried under a lamp. The catalyst slurry was made by sonicating the catalyst $(1 \mathrm{mg})$ in a $3: 1$ water-isopropanol mixture $(1 \mathrm{~mL})$ containing $10 \mu \mathrm{L}$ of Nafion solution (5 wt $\%$ ) and was drop-coated onto a previously polished GCE to afford an uniform thin film with a constant loading of $102 \mu \mathrm{g} \mathrm{cm} \mathrm{cm}^{-2}$ in all cases. The electrode was dried at room temperature in air without any heating process and was used as the WE for all further electrochemical studies. An aqueous solution of $0.1 \mathrm{M} \mathrm{KOH}$, which had been de-aerated with $\mathrm{N}_{2}$ or $\mathrm{O}_{2}$, was used as an electrolyte for the normal $\mathrm{CV}$ and RDE/RRDE studies. Prior to the electrochemical analysis, the electrode surface was electrochemically cleaned by repeatedly cycling the potential between $-\mathbf{1 . 0}$ and $0.2 \mathrm{~V}$ for more than 20 cycles at a scan rate of $100 \mathrm{mV} \mathrm{s}^{-1}$. For the $\mathrm{MeOH}$ crossover study, a $3 \mathrm{M}$ solution of $\mathrm{MeOH}(100 \mathrm{~mL})$ was used as the electrolyte, with $0.1 \mathrm{M} \mathrm{KOH}$ as the supporting electrolyte. Plots were normalized by dividing the measured currents by the geometric area of the WE. In order to exclusively analyze the ORR current, the RDE/RRDE polarization curves measured from $\mathrm{O}_{2}$-saturated solution were corrected by subtracting background surface oxidation and capacitive processes measured from $\mathrm{N}_{2}$-saturated solution. All experiments were conducted at ambient temperature $\left(25 \pm 2{ }^{\circ} \mathrm{C}\right)$.

\section{Results and discussion}

\section{Microstructure analysis on NGPCs}

A schematic diagram describing the structural reorganization of ZIF-8 NCs to nanoporous carbon NP is shown in Scheme 1. The ZIF-8 parent model has a cage-type pore structure with large pore cavities of $11.6 \AA$ (indicated by the yellow spheres) connected by its small pore apertures of $3.4 \AA$ and features a rhombic dodecahedral morphology. After single-step direct carbonization, it was converted into nitrogen-doped graphitic porous carbon, retaining its original morphology. PXRD profiles and first-order Raman spectra of the typical NGPCs obtained from $1000{ }^{\circ} \mathrm{C}$ are shown in Fig. 1a and b, respectively. All the materials displayed only two broad peaks located at around $25^{\circ}$ and $44^{\circ}$ that were assigned to the characteristic carbon (002) and $(100) /(101)$ diffractions, respectively, revealing the presence of long-range two-dimensional ordering in the carbon matrices 

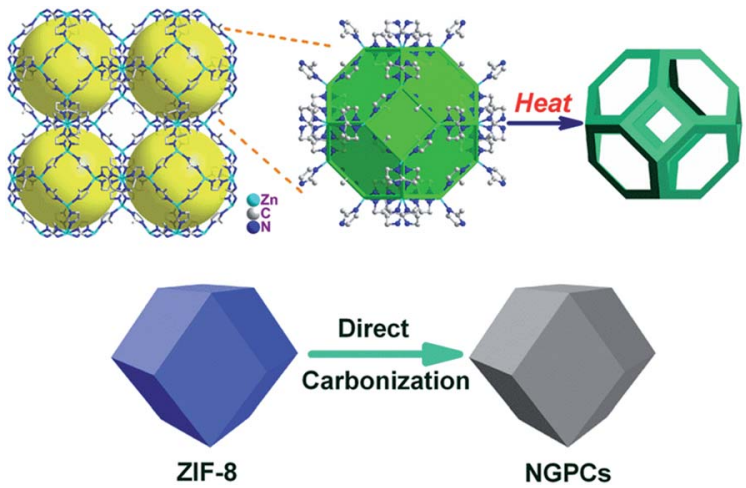

Scheme 1 Schematic illustration of the NMOFs-driven template synthesis of highly graphitized nitrogen-doped porous carbon nanopolyhedra.

along with some graphitization. A large intensity increase in the low-angle scatter shows the presence of a high density of nanopores. No significant change in the full width at half maximum (FWHM) of the (002) and (100)/(101) reflections indicates the similar crystallite size of the samples. Additionally, no diffraction peaks of impurities could be observed suggesting the complete conversion of the carbonaceous materials after the heat treatment of the ZIF-8 NCs. In the carbonization process the $\mathrm{Zn}$ ions were reduced to $\mathrm{Zn}$ metals (boiling point $908{ }^{\circ} \mathrm{C}$ ) by carbon and then vaporized under the $T_{\mathrm{c}}$ of $1000{ }^{\circ} \mathrm{C}$, and any residual was further leached out by the following acid wash. The ICP analysis validated the absence of traceable $\mathrm{Zn}$ species in the NGPCs (not shown). Moreover, the relatively stronger and slight positive shift of the (002) peak in the PXRD profile of NGPC-1000-10, indicates its higher degree of graphitization. The PXRD diagrams of the NGPCs at various $T_{\mathrm{c}}$ (Fig. S1†) depict that the framework of ZIF-8 NCs was stable up to $500{ }^{\circ} \mathrm{C}$, then partially decomposed at $600{ }^{\circ} \mathrm{C}$ and were fully converted to carbons (i.e., $\mathrm{CNx}$ ) at $700{ }^{\circ} \mathrm{C}$, which was further confirmed by the TGA curve of ZIF-8 (Fig. S2 $\dagger$ ). As shown in the Raman spectra, a typical D band roughly at $1350 \mathrm{~cm}^{-1}$ resulting from the in-plane imperfections such as defects and heteroatoms of the graphitic lattice of the disordered $\mathrm{sp}^{2}$-hybridized carbon, and a $\mathrm{G}$ band at $1585 \mathrm{~cm}^{-1}$ from the tangential
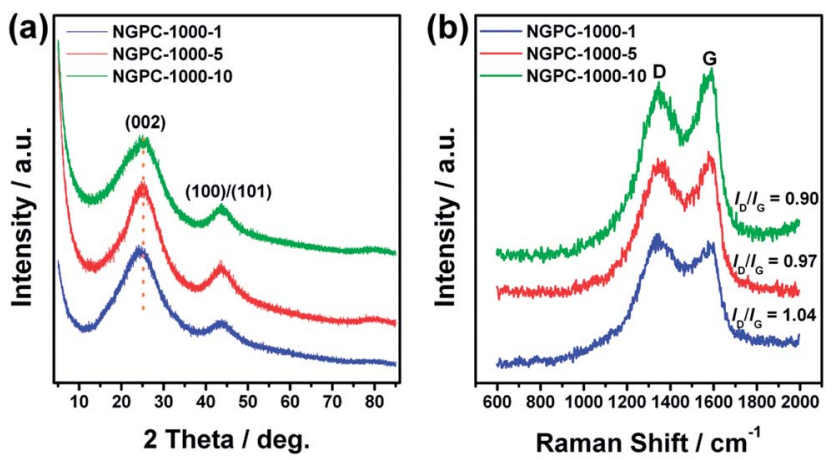

Fig. 1 Representative (a) wide-angle $\mathrm{X}$-ray diffraction patterns, (b) Raman spectra of NGPCs obtained at $1000^{\circ} \mathrm{C}$. stretching mode of highly ordered pyrolytic graphite, indicating the presence of crystalline graphitic carbon, ${ }^{43}$ could be observed for all samples. The sample to sample variation of the relative intensity ratio of $\mathrm{D} / \mathrm{G}$ bands $\left(I_{\mathrm{D}} / I_{\mathrm{G}}\right)$ from $0.90-1.04$ suggests that longer carbonization time generates slightly more turbostratic/ graphitic carbons, i.e., higher degree of graphitization. The Raman spectra of samples prepared at other $T_{\mathrm{c}}$ are shown in Fig. S3. $\dagger$ Generally, the $I_{\mathrm{D}} / I_{\mathrm{G}}$ increased with increasing temperature, and both the $\mathrm{D}$ and $\mathrm{G}$ peaks became better resolved with narrower and sharper peaks and with an increased distance between the two peaks. This trend implied that fewer defects and more graphitic crystallites were present at higher $T_{\mathrm{c}}$. The high degree of graphitization will surely improve the electrical conductivity of the NGPCs, which is beneficial to enhance the ORR activity.

\section{Microscopic analysis on NGPCs}

To authenticate the effect of morphology and porosity of the parent ZIF-8 NCs on the resulting carbons, SEM and TEM characterization were conducted. The geometric shape of the asprepared ZIF-8 NCs were demonstrated as rhombic dodecahedra with an average diameter around $60 \mathrm{~nm}$ (Fig. 2a and b), consistent with previous reports. ${ }^{44}$ For better visualization of the polyhedral shape of the ZIF-8 NCs, a schematic illustration of the rhombic dodecahedron with 12 exposed $\{110\}$ facets is given in Fig. 2c. Correspondingly, typical TEM images of individual ZIF-8 and the resulting NGPC are also shown in Fig. $2 \mathrm{~d}$ and e, respectively. The original shape of ZIF-8 NCs was perfectly retained in the NGPC after high temperature carbonization. The gray color with lighter contrast of NGPC compared to ZIF-8 is due to its composition of lighter elements of $\mathrm{C}$ and $\mathrm{N}$ (low $Z$ value). In addition to the polyhedral morphology, NGPCs appeared to preserve the structural integrity and monodispersity as well, with a slightly decreased size and crumpled surface as expected (Fig. $2 \mathrm{f}$ and $\mathrm{g}$ ). Generally, the shape gradually shrunk with the increase of $T_{\mathrm{c}}$ as well as the carbonization time. At last, a certain fraction of carbons with totally open frameworks were formed, accompanied by a rougher surface and seriously anisotropic shrink of MOF structures (Fig. S4-S6†). The selected-area electron diffraction (SAED) pattern (inset in Fig. 2g) shows clear diffraction rings, suggesting the formation of graphitic pore walls. The pore structure of NGPCs was confirmed by the observation of distinct nanovoids distributed over the entire area of a single particle shown in Fig. 2h. Moreover, the high resolution TEM image of NGPCs (Fig. 2i) further indicated the formation of crystalline frameworks and that oriented multilayer domains and a few indistinguishable graphene sheets stacked in parallel positions, as evident from the regularly spaced dark lines with adjacent interlayer distances of $0.3-0.4 \mathrm{~nm}$, close to the $d$-spacing of the (002) crystal plane $(0.335 \mathrm{~nm})$ of bulk graphite with slight distortion, ${ }^{45}$ coinciding with the PXRD results. The nanoporous walls consist of local graphitic structure, which can potentially benefit ORR electrocatalysis by enhancing the corrosion resistance and electrical conductivity. From the TEM images of NGPCs, it is clear that the structural arrangement or rigidity of MOF architecture is a responsible factor for the formation of 

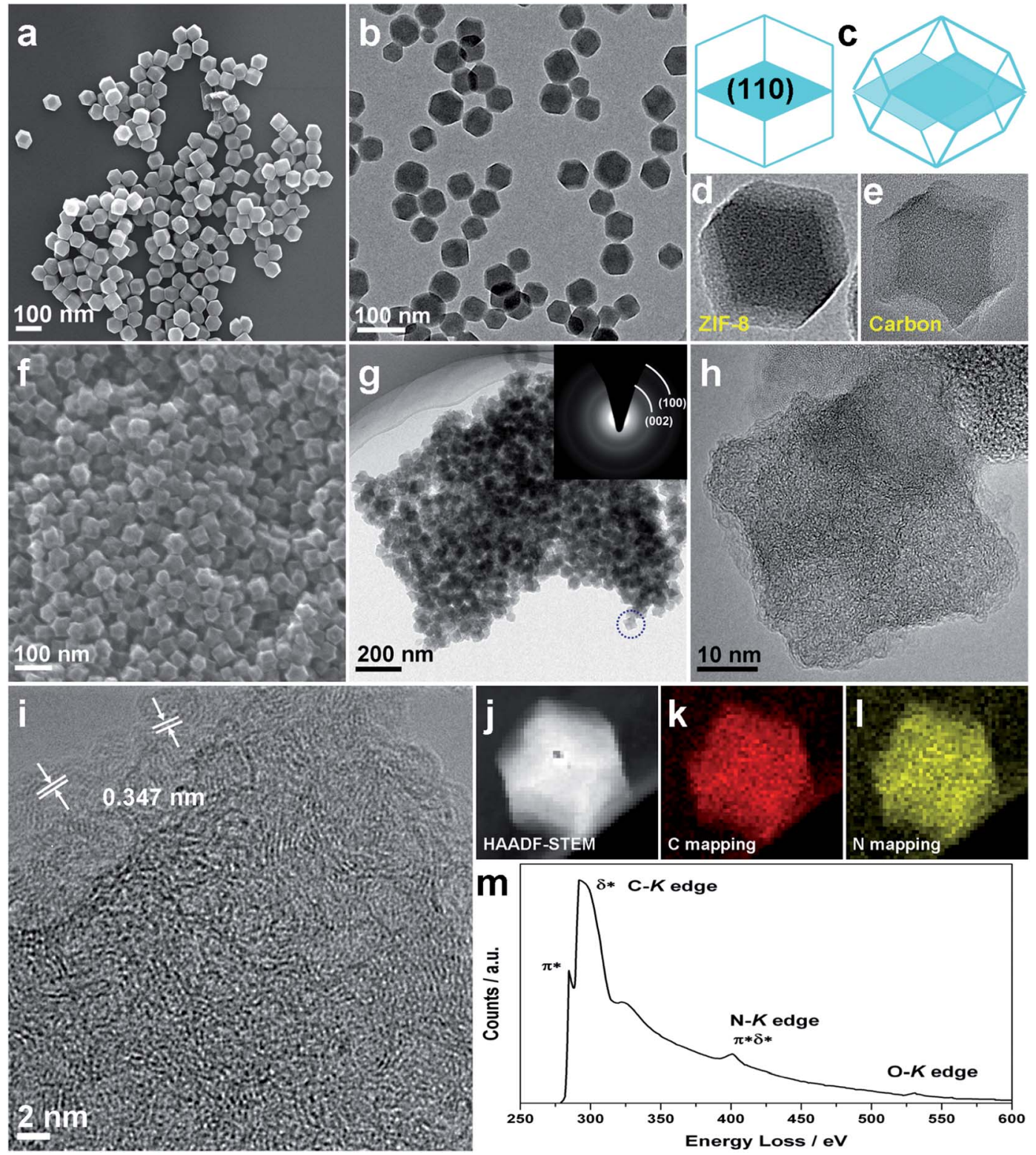

Fig. 2 (a) SEM, (b) TEM images of mono-dispersive ZIF-8 nanopolyhedra; and (c) the corresponding rhombic dodecahedron-like structural models. (d) and (e) TEM images of typical ZIF-8 and NGPC polyhedron nanoparticle, respectively. (f) SEM and (g) TEM images of NGPC-1000-10; inset in (g) shows the corresponding SAED pattern. (h) TEM image of a single NGPC polyhedron taken from the circular region marked in (g). (i) HRTEM images taken at the corner of the NGPC particle shown in (h). (j) to (l) show the HAADF-STEM images of a single carbon polyhedron and the corresponding $\mathrm{C}-$, and $\mathrm{N}$-elemental mappings. (m) Electron energy loss spectrum (EELS) of NGPC-1000-10.

short-range ordered pores in the carbon matrix. It is widely believed that, during the decomposition of MOFs, the structure collapse at elevated temperature restricts its use as a true hard template for carbon formation. However, as evident from the TEM images of NGPCs (Fig. S7†), one could still observe the presence of pores along with the retention of original MOF morphology with few exceptions, which substantiates the role of ZIF-8 as an ideal template for carbonization. The elemental composition analysis from the energy dispersive spectrum (EDS) pattern confirmed the existence of $\mathrm{N}$ in NGPCs (Fig. S8 $\dagger$ ). This result was further supported by the relative uniform $\mathrm{N}$ distribution in the plane of NGPCs verified by the high-angle annular dark field scanning-
TEM (HAADF-STEM) and corresponding element mapping images on a single NGPC particle (Fig. $2 \mathrm{j}-\mathrm{l}$ ). Furthermore, the $1 \mathrm{~s}-$ $\pi^{*}$ and $\pi-\pi^{*}$ transition peaks at 284 and $401 \mathrm{eV}$ in the K-edges of $\mathrm{C}$ and $\mathrm{N}$ atoms observed by electron energy loss spectrum (EELS) in Fig. 2m, respectively, indicates that nitrogen atoms were rather well hybridized into the carbon $\mathrm{sp}^{2}$ aromatic rings. Elemental analysis revealed the following composition (wt\%) of NGPC-100010: C 88.01, H 2.40, N 4.66, O (calculated) 4.93.

\section{Textural architectures in NGPCs}

The presence of nanopores in NGPCs was further confirmed by nitrogen sorption measurement. The isotherms and pore size 

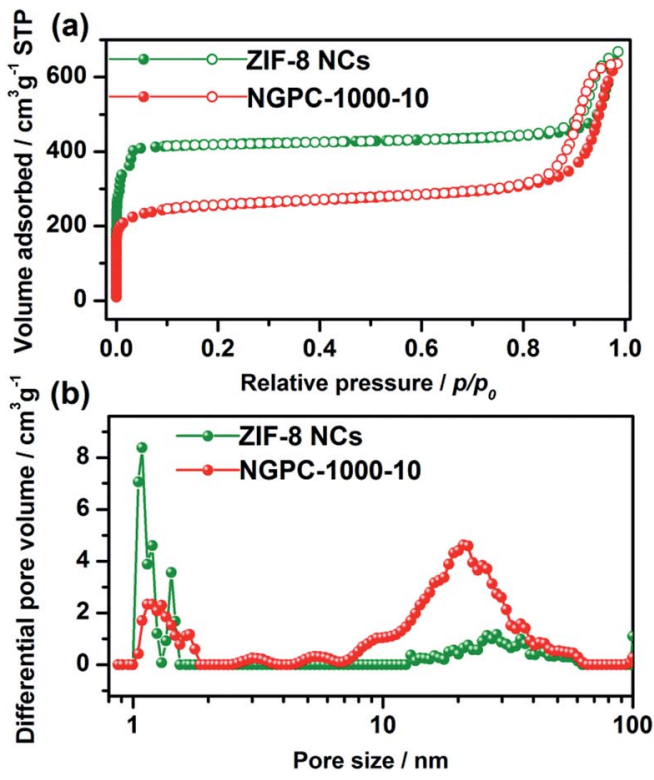

Fig. 3 Comparative (a) nitrogen sorption isotherms at $77 \mathrm{~K}$ (closed, adsorption; open, desorption) and (b) the corresponding NL-DFT pore size distribution curves of ZIF-8 nanocrystals and NGPC-1000-10.

distribution (PSD; derived using the non-local density functional theory (NL-DFT) method) of NGPC-1000-10 are shown in Fig. 3 and the textural properties of all the NGPCs are summarized in Table 1. The NGPC-1000-10 showed typical Type-I isotherms with sharp uptakes at low relative pressure $\left(P / P_{0}<\right.$ $0.015)$ but a large hysteresis loop at high relative pressure $\left(P / P_{0}>\right.$ 0.85 ), suggesting the intrinsic micropore characteristic with a portion of meso/macropores caused by the sorption of internanoparticle voids, the presence of which was not observed in the corresponding bulk ZIF-8 derived porous carbons and should be considered as additional second-order structures beneficial for efficient mass transport and smooth diffusion of electrolyte and oxygen during the electrocatalytic reactions. The resulting enhanced ORR activity suggests the advantage of using nanoscale ZIF-8 as a precursor in the present work. ${ }^{46}$ The $\mathrm{N}_{2}$ sorption profile of NGPC-1000-10 is similar to that of the parent ZIF-8 NCs but with a reduced sorption amount and a more pronounced hysteresis feature, indicating more mesoporous cavities in the more rigid carbon structure, presumably the result of overall dimensional shrinkage and the coagulated nature of the carbonization products. More importantly, the PSD analysis of NGPC-1000-10 disclosed the similarity in pore size at the microporous diameter region $(1-2 \mathrm{~nm})$ with that of ZIF-8 NCs, showing a typical diameter centered around $12 \AA$, close to the larger of the two kinds of pores (3.4 and $11.6 \AA$ A) in the parent ZIF-8. This further indicates that the carbon materials obtained via direct carbonization of MOFs could possess a pore diameter similar to that of their parent MOFs, in addition to the similar crystal morphology. Undoubtedly, the highly regular MOF structure with prearranged distribution of organic units should, in principle, afford carbon materials with persistent atomic arrangements, as well as reproducible and well-controllable properties. Furthermore, a relatively much higher ratio of meso/macropores to micropores $\left(V_{\text {meso+macro }} / V_{\text {micro }}\right)$ was observed for NGPC$1000-10$ in the pore diameter range of 7-60 nm with peak-top located at $20 \mathrm{~nm}$, further supporting the aforementioned analysis for $\mathrm{N}_{2}$ sorption. The hierarchically porous NGPC1000-10 possesses a total pore volume of $0.99 \mathrm{~cm}^{3} \mathrm{~g}^{-1}$ and $V_{\text {meso+macro }} / V_{\text {micro }}$ volume ratio of 2.09 , which are $c a .2 .7$ and 3.5 times higher than that of their counterparts derived from bulk ZIF-8, respectively, suggesting the enhanced porous features are attributed to the nanoscale dimension of the nanopolyhedral carbons. ${ }^{46}$ The $\mathrm{N}_{2}$ sorption isotherms of the samples obtained at different $T_{\mathrm{c}}$ generally showed a continuous increase in sorption with increasing $T_{\mathrm{c}}$ (Fig. S9†). However, their corresponding PSD curves are similar to each other but with a gradual shift of the peaks in the meso/macropore region to lower pore diameter (Fig. S10 $\dagger$ ), confirming the further shrinkage and agglomeration of NGPCs at higher temperature. As seen in Table 1, from 700 to $900{ }^{\circ} \mathrm{C}$, reasonable to high SSA ranging from 416 to $1076 \mathrm{~cm}^{3} \mathrm{~g}^{-1}$ were obtained for $5 \mathrm{~h}$ carbonization. However, the SSA then slightly decreased to $973 \mathrm{~cm}^{3} \mathrm{~g}^{-1}$ at $1000{ }^{\circ} \mathrm{C}$, probably owing to the cleavage of $\mathrm{C}-\mathrm{N}$ and escape of nitrogen as well as the further graphitization of the carbon framework at higher temperature. ${ }^{47}$ It is also worth mentioning that the acid leaching is important for the purification of the products, as NGPC-700-5 generally exhibited a nonporous character with a low SSA of only $106 \mathrm{~cm}^{3} \mathrm{~g}^{-1}$ before

Table 1 Pore characteristics of the ZIF-8 NCs and the resultant NGPCs

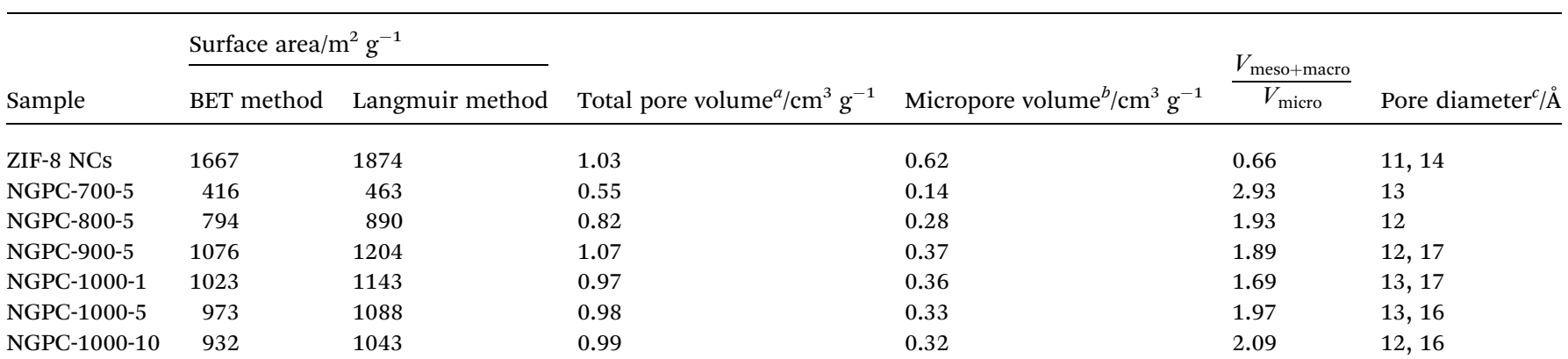

${ }^{a}$ At $P / P_{0}=0.99 .{ }^{b}$ Determined by $t$-plot method. ${ }^{c}$ Determined by NL-DFT method and the listed pore diameter refers to the pore size corresponding to the peak position at the micropore range $(<2 \mathrm{~nm})$. 
leaching (Fig. S11 $\dagger$ ), while this value significantly increased to $416 \mathrm{~cm}^{3} \mathrm{~g}^{-1}$ after the removal of $\mathrm{Zn}$ impurities.

\section{Nitrogen-doping analysis on NGPCs}

In the growth of ZIF-8 NCs, excess MeIM ligands act as terminating linker units in their protonated form ${ }^{48}$ thus forming substantial $\mathrm{C}-\mathrm{N} / \mathrm{C}=\mathrm{N}$ moieties on the surface of the carbon materials after carbonization, where the catalytic oxygen reduction occurs. To probe the nitrogen atoms in the NGPCs structures, XPS measurements were carried out. As displayed in Fig. 4a, the XPS survey spectrum for the NGPC-1000-10 showed a predominant narrow graphitic C1s peak at $285 \mathrm{eV}$, along with a N1s peak at $c a .400 \mathrm{eV}$. Additionally, an O1s peak at $c a .540 \mathrm{eV}$ was also observed, probably due to the physically adsorbed oxygen or water as in the case of most nitrogen-doped CNTs. ${ }^{10,49}$ The absence of any $\mathrm{Zn}$ peak on the survey spectrum clearly indicates that the $\mathrm{Zn}$ residues, if any, were completely removed by the $\mathrm{H}_{2} \mathrm{SO}_{4}$ solution. The high resolution N1s spectrum (Fig. 4b) can be deconvoluted to four sub-peaks due to the spinorbit coupling, including pyridinic- $\mathrm{N}(\mathrm{N} 1,398.4 \pm 0.1 \mathrm{eV})$, pyrrolic-N (N2, $399.8 \pm 0.1 \mathrm{eV})$, graphitic-N $(\mathrm{N} 3,400.9 \pm 0.1 \mathrm{eV})$ and pyridine-N-oxide groups $(\mathrm{N} 4,402.4 \pm 0.3 \mathrm{eV}) .{ }^{39}$ The deconvoluted C1s signal in Fig. 4c showed a slightly asymmetric tail at higher binding energy, which is a common characteristic for nitrogen-doped carbon materials. ${ }^{49}$ The two main peaks at 284.6 and $285.7 \mathrm{eV}$ were assigned to $\mathrm{sp}^{2}$-hybridized graphite-like carbon (C-C sp ${ }^{2}$ ) and $\mathrm{sp}^{3}$-hybridized diamond-like carbon (C-C $\mathrm{sp}^{3}$ ), respectively. ${ }^{50}$ The peaks centered at 286.6, 287.6 and $289.1 \mathrm{eV}$ were attributed to surface oxygen and nitrogen groups (designated as $\mathrm{C}-\mathrm{O} / \mathrm{C}-\mathrm{N}, \mathrm{C}=\mathrm{O} / \mathrm{C}=\mathrm{N}$ and $\mathrm{O}=\mathrm{C}-\mathrm{O}$, respectively). ${ }^{19}$ And the appearance of $\pi-\pi^{*}$ shake up satellites of $\mathrm{sp}^{2}$ graphite-like carbon above $290 \mathrm{eV}$ indicates the further
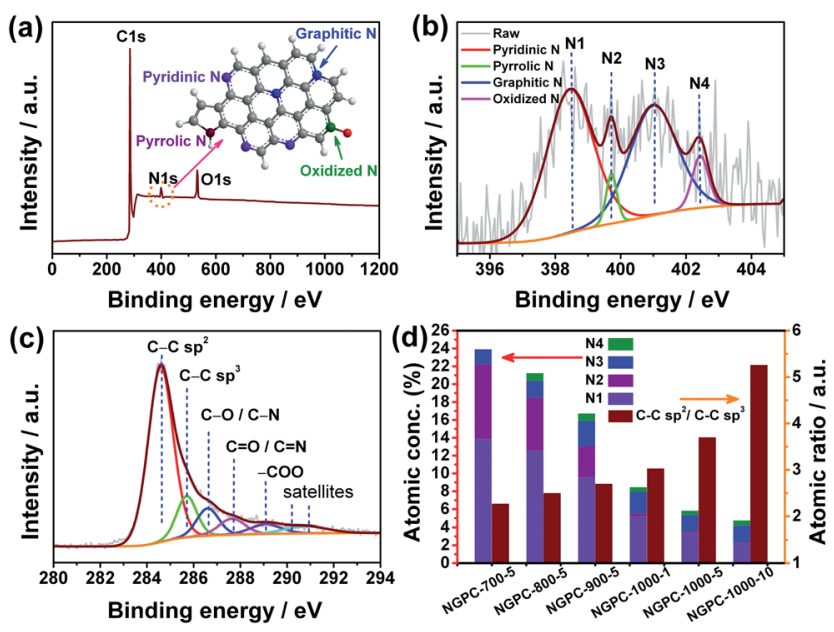

Fig. 4 Typical (a) survey scanned XPS spectrum; (b) deconvoluted N1s spectrum and (c) deconvoluted C1s spectrum of NGPC-1000-10; the inset in (a) shows the schematic illustration of the four kinds of chemical bonding configurations of nitrogen dopant (pyridinic- $N, N 1$; pyrrolic-N, N2; graphitic-N, N3; and oxidized-N, N4). (d) Bar diagrams representing the variation of the atomic surface concentration of the four kinds of nitrogen species and the atomic ratio of $\mathrm{C}-\mathrm{C} \mathrm{sp} / \mathrm{C}-\mathrm{C}$ $\mathrm{sp}^{3}$ in different NGPCs, respectively. carbonization and aromatization during the prolonged carbonization time of NGPC-1000-10. ${ }^{50}$ The similar deconvolution of N1s and C1s peaks of other NGPCs obtained at various $T_{\mathrm{c}}$ and times were also executed (Fig. S12 and S13†), and information related to the type and content of the nitrogen species present in the NGPCs is presented in Fig. $4 \mathrm{~d}$ and summarized in Table S1 $\uparrow$ as well. It was found that with increasing carbonization temperature from 700 to $1000{ }^{\circ} \mathrm{C}$ under the same $5 \mathrm{~h}$ carbonization, the total $\mathrm{N}$ content decreased from 23.9 to 5.82 at $\%$, and yet is still among the highest doping levels reported in the literature. ${ }^{21}$ The sharp decrease of $\mathrm{N}$ content above $900{ }^{\circ} \mathrm{C}$ might be a consequence of breakage of the $\mathrm{C}-\mathrm{N}$ bond, since it might decompose quickly at the temperature over $850{ }^{\circ} \mathrm{C} .{ }^{47}$ Furthermore, the pyridinic- $\mathrm{N}$ is predominant for all the samples with slightly varied relative content (57-59\%), which agrees with the results of other studies that the formation of pyridinic- $\mathrm{N}$ is preferred at higher overall nitrogen content. ${ }^{49}$ The relative content of graphitic-N progressively increased with the increasing temperature, whereas the pyrrolic- $\mathrm{N}$ gradually decreased from 35 to $21 \%$ with the $T_{\mathrm{c}}$ increasing from 700 to $900{ }^{\circ} \mathrm{C}$, and then quickly diminished at $1000{ }^{\circ} \mathrm{C}$, achieving a negligible portion of $c a .2 \%$ of the total N in NGPC-1000-10. This is due to its thermally unstable nature and easy conversion to graphitic-N at higher temperature. Notably, the sum portion of pyridinic- and graphitic-type nitrogen in the samples prepared at $1000{ }^{\circ} \mathrm{C}$ almost remained constant ( $c a .90 \%$ of the total $\mathrm{N}$ ), indicating a continuous conversion from pyridinic- $\mathrm{N}$ to graphitic-N at this temperature. ${ }^{39}$ The results suggest that this simple MOF-driven synthetic strategy can easily control the nitrogen doping level by adjusting the carbonization temperature and time. Moreover, Fig. $4 \mathrm{~d}$ also shows that with increasing $T_{\mathrm{c}}$, a noticeable increase of the atomic ratio of $\mathrm{C}-\mathrm{C} \mathrm{sp}^{2} / \mathrm{C}-\mathrm{C} \mathrm{sp}{ }^{3}$ was obtained. This observation is in good agreement with the Raman results, disclosing that the major fraction of carbon species in NGPCs is $\mathrm{sp}^{2}$-hybridized owing to the aromatic nature of MeIM ligands in the ZIF-8 NCs precursor, thus rendering the carbonaceous materials electrical conducting. FTIR spectroscopic measurement also indicates the formation of condensed $\mathrm{C}-\mathrm{N} / \mathrm{C}=\mathrm{N}$ bonds, along with the presence of $\mathrm{N}-\mathrm{H}$ and $\mathrm{C}-\mathrm{O}$ functional groups which endow NGPCs with an active surface for improved hydrophilicity and wettability facilitating the oxygen access to the catalyst surface in the ORR process (Fig. S14†).

\section{Electrocatalytic performance of NGPCs for ORR}

The electrocatalytic activities of NGPCs for ORR were first scrutinized by $\mathrm{CV}$ in $0.1 \mathrm{M} \mathrm{KOH}$ solution in the absence $\left(\mathrm{N}_{2}\right.$ bubbling) and presence of oxygen $\left(\mathrm{O}_{2}\right.$ bubbling) at room temperature (Fig. S15†). Typically, as shown in Fig. 5a, quasirectangular voltammograms with featureless voltammetric currents within the potential range from -1.0 to $0.2 \mathrm{~V}(\mathrm{vs}$. Ag/ $\mathrm{AgCl}$ ) were observed in the $\mathrm{N}_{2}$-saturated solution for samples prepared at $1000{ }^{\circ} \mathrm{C}$, as a result of the typical supercapacitance effect on porous carbon materials, suggesting that any metal residuals that might have remained from the NGPCs synthesis process were completely removed from the NGPCs surface, thus 

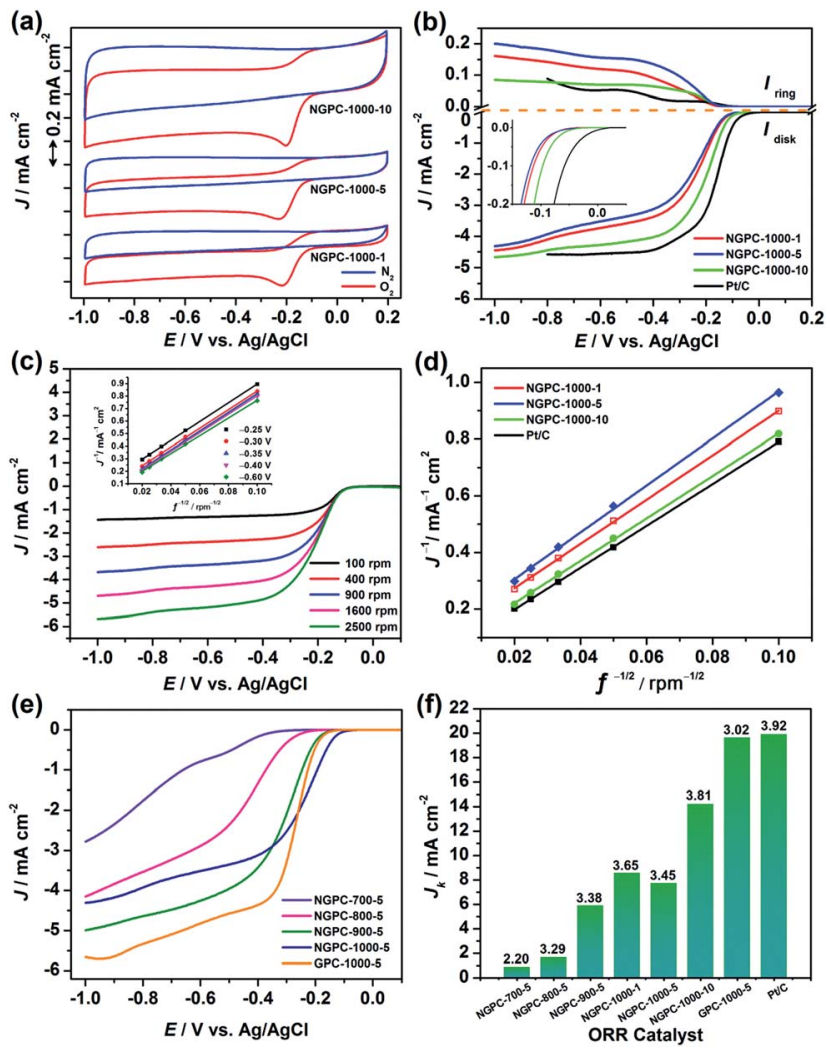

Fig. 5 (a) Cyclic voltammograms of NGPCs obtained at $1000^{\circ} \mathrm{C}$ with a scan rate of $10 \mathrm{mV} \mathrm{s}^{-1}$ in $\mathrm{O}_{2}$ - (red) and $\mathrm{N}_{2}$-saturated (blue) $0.1 \mathrm{M} \mathrm{KOH}$ solution. (b) Linear sweep voltammograms (LSVs) of NGPCs obtained at $1000{ }^{\circ} \mathrm{C}$ and $20 \mathrm{wt} \% \mathrm{Pt} / \mathrm{C}$ in $\mathrm{O}_{2}$-saturated $0.1 \mathrm{M} \mathrm{KOH}$ solution at $1600 \mathrm{rpm}$ with a scan rate of $5 \mathrm{mV} \mathrm{s}^{-1}$ after background subtraction (inset: magnification of the potential region of the ORR onset). (c) LSVs of NGPC-1000-10 in $\mathrm{O}_{2}$-saturated $0.1 \mathrm{M} \mathrm{KOH}$ with various rotation speeds at a scan rate of $5 \mathrm{mV} \mathrm{s}^{-1}$. The corresponding Koutecky-Levich $(\mathrm{K}-\mathrm{L})$ plots at various voltages are shown in the inset. (d) Comparative $\mathrm{K}-\mathrm{L}$ plots of NGPCs obtained at $1000^{\circ} \mathrm{C}$ and $20 \mathrm{wt} \% \mathrm{Pt} / \mathrm{C}$ at $-0.35 \mathrm{~V}$ derived from the corresponding LSVs. (e) LSVs of NGPCs obtained at different temperatures with a carbonization time of $5 \mathrm{~h}$ in $\mathrm{O}_{2}$-saturated $0.1 \mathrm{M} \mathrm{KOH}$ at $1600 \mathrm{rpm}$ with a scan rate of $5 \mathrm{mV} \mathrm{s}^{-1}$. (f) Electrochemical activity given as the kinetic current density $\left(J_{K}\right)$ at $-0.35 \mathrm{~V}$ for all samples, the numeral on the bar represents the corresponding electron transfer number.

not contributing to the ORR activity. The CVs in $\mathrm{O}_{2}$-saturated solution, in contrast, exhibited a steep rise in the oxygen reduction current, reaching a well-defined cathodic peak for all sample electrodes, hence suggesting a pronounced electrocatalytic activity of NGPCs for oxygen reduction. Generally, the peak potentials of various NGPCs positively shifted to higher voltage as the $T_{\mathrm{c}}$ increased. Furthermore, with the carbonization time of NGPCs increasing, the peak potentials clearly shifted from -0.22 (NGPC-1000-1) to -0.23 (NGPC-1000-5) and $-0.20 \mathrm{~V}$ (NGPC-1000-10), accompanied by a progressively increased maximum peak current density from 0.49 (NGPC-1000-1) to 0.54 (NGPC-1000-5) and $0.80 \mathrm{~mA} \mathrm{~cm}{ }^{-2}$ (NGPC-1000-10). The more positive peak potential and higher reduction current density of NGPC-1000-10 for ORR indicates the highest activity among the NGPCs obtained at $1000{ }^{\circ} \mathrm{C}$. Surprisingly, NGPC-1000-5 exhibited a peak potential of $10 \mathrm{mV}$ negatively shifted to that of
NGPC-1000-1, although its peak current density was slightly higher, suggesting that longer carbonization time does not usually result in higher ORR activity.

The impressive electrocatalytic activity of NGPC-1000-10 was further confirmed by recording LSV curves on a RRDE. As shown in Fig. 5b, the current density simultaneously increased as the potential became more negative, which is commonly observed on metal-free ORR catalysts with mesopores. ${ }^{12}$ For comparison, NGPC-1000-1, NGPC-1000-5 and commercial $20 \mathrm{wt} \% \mathrm{Pt} / \mathrm{C}$ were also investigated under the same conditions. It was found that similar evolutions were also observed for the limiting current densities $\left(J_{\mathrm{L}}\right)$, onset potentials $\left(E_{\text {onset }}\right)$ and half-wave potentials $\left(E_{1 / 2}\right)$ of NGPCs as the peak potentials in CV measurements. Again, NGPC-1000-10 exhibited the highest electrocatalytic activity for the ORR, as reflected by its higher positively shifted $E_{\text {onset }}$ and higher current density of $4.3 \mathrm{~mA} \mathrm{~cm}^{-2}$ than that of NGPC-1000-1 (3.7 mA cm $\left.{ }^{-2}\right)$ and NGPC-1000-5 $\left(3.5 \mathrm{~mA} \mathrm{~cm}^{-2}\right)$ at $-0.60 \mathrm{~V}$, which is close to the corresponding value of $\mathrm{Pt} / \mathrm{C}(4.5$ $\left.\mathrm{mA} \mathrm{cm}^{-2}\right)$. Remarkably, both the $E_{\text {onset }}(-0.02 \mathrm{~V})$ and $E_{1 / 2}(-0.20$ V) of NGPC-1000-10 are only less than $40 \mathrm{mV}$ negatively shifted than those of Pt/C (inset of Fig. 5b).

To gain further insights regarding the reaction kinetics and the number of electrons transferred per oxygen molecule on NGPCs, RDE measurements at different rotating speeds from 100 to $2500 \mathrm{rpm}$ were conducted (Fig. S16 $\dagger^{\dagger}$ ). In the ORR process, oxygen within the solution diffuses to the surface of the electrode and reacts to form water preferably through a four-electron pathway, or to produce $\mathrm{H}_{2} \mathrm{O}_{2}$ undesirably through a twoelectron pathway. The electron transfer number $(n)$ and the kinetic current density $\left(J_{\mathrm{K}}\right)$ can be calculated based on the Koutecky-Levich (K-L) equation (see the ESI $\dagger$ ). As shown in Fig. 5c, the LSV curves of the NGPC-1000-10 at different rotation speeds clearly reflect that the $J_{\mathrm{L}}$ increased with increasing rotation speed due to the reduced diffusion distance and the corresponding $\mathrm{K}-\mathrm{L}$ plots $\left(J^{-1}\right.$ vs. $\left.f^{-1 / 2}\right)$ at various potentials (inset of Fig. 5c) exhibited good linearity and parallelism, suggesting first-order reaction kinetics with respect to the concentration of dissolved $\mathrm{O}_{2}$. The slopes remained approximately constant over the potential range from -0.25 to $-0.60 \mathrm{~V}$, which suggests that the $n$ values for oxygen reduction at different potentials are similar. The $n$ value is calculated to be 3.81 for NGPC-1000-10 at $-0.35 \mathrm{~V}$, close to the highly-desired four-electron ORR pathway of maximum energy capacity. Meanwhile, the corresponding $J_{\mathrm{K}}$ value of $14.2 \mathrm{~mA} \mathrm{~cm}^{-2}$ is also comparable to that of $\mathrm{Pt} / \mathrm{C}\left(19.9 \mathrm{~mA} \mathrm{~cm}{ }^{-2}\right)$, which can be observed by the similar slopes and intercepts of the comparative K-L plots of NGPC-1000-10 and Pt/C (Fig. 5d). The nearly linear regression of the K-L plots for NGPC-1000-1 and NGPC-1000-5 compared with NGPC-1000-10 clearly indicates their inferior kinetic activity. Their $n$ values calculated at $-0.35 \mathrm{~V}$ reach 3.65 and 3.45, respectively, indicating that their ORR both proceeds predominantly with a one-step, four-electron process. However, the $n$ values for NGPC-1000-10 are more stable and higher at 3.80-3.89 over most of the potential range (Fig. S17†), indicating a smoother and more electrochemically stable ORR process over NGPC-1000-10. The corresponding much more smoothly increased ring current density as the potential became more 
negative confirmed this result (Fig. 5b), and also revealed the high selectivity of $91 \%$ of the preferred $\mathrm{H}_{2} \mathrm{O}$ product on the NGPC-1000-10 electrode. We compared the ORR performance of NGPC-1000-10 with other electrocatalysts, as shown in Table S2. $\dagger$ The results demonstrated here for NGPC-1000-10 are superior than or similar to previously reported N-doped CNTs or graphene electrocatalysts in alkaline media.

Since the NGPCs fabricated from the ZIF-8 NCs precursor originated from a carbonization process followed by an acid wash, the morphology and pore texture of the parent MOF were well inherited and even enhanced, which enables a firm correlation between their mesostructural characteristics and electrochemical performance. Thus, various versions of NGPCs prepared at different $T_{\mathrm{c}}$ (i.e., 700, 800, 900, $1000^{\circ} \mathrm{C}$ ) were further investigated. Their RDE voltammograms in $\mathrm{O}_{2}$-saturated $0.1 \mathrm{M}$ $\mathrm{KOH}$ solution at a rotating speed of $1600 \mathrm{rpm}$ were compared in Fig. 5e, and the derived results are outlined in Table 2. Specifically, a higher current density was obtained for samples prepared at higher $T_{\mathrm{c}}$ when temperature was raised from 700 to $900{ }^{\circ} \mathrm{C}$, associated with a higher $E_{\text {onset }}$ and $E_{1 / 2}$. Notably, the current density of NGPC-900-5 below $-0.35 \mathrm{~V}$ even exceeded that of NGPC-1000-5. However, NGPC-1000-5 displayed the highest $E_{\text {onset }}$ and $E_{1 / 2}$ among these samples. The NGPC-700-5 showed a two-step process for ORR, with the $E_{\text {onset }}$ values at -0.21 and $-0.54 \mathrm{~V}$, respectively. The calculated average $n$ value is 2.12. This result suggests that its reduction process favors a two-electron over a four-electron pathway with a poor selectivity of $\mathrm{H}_{2} \mathrm{O}(12 \%)$. While the oxygen reduction on other electrocatalysts may proceed in a coexisting pathway involving both the two-electron and four-electron transfers with average $n$ values and $\mathrm{H}_{2} \mathrm{O}$ selectivity ranging from 3.44 to 3.52 and 65.0 to $75.5 \%$, respectively. The $J_{\mathrm{K}}$ values of these samples at $-0.35 \mathrm{~V}$ also gradually increased as the $T_{\mathrm{c}}$ increased, ranging from 0.86 (NGPC-700-5) to $7.70 \mathrm{~mA} \mathrm{~cm}^{-2}$ (NGPC-1000-5), as shown in Fig. 5f. The much inferior kinetic activity of NGPC-700-5 and NGPC-800-5 can also be observed from their sharply increased current densities in the potential range below $-0.40 \mathrm{~V}$, which are far from the reach of current plateaus. For comparison, a nitrogen-free graphitic porous carbon derived from a wellknown MOF-5 material (denoted as GPC-1000-5) was also studied. Compared with NGPC-1000-5, GPC-1000-5 has a much higher degree of graphitization and 2.4 times higher SSA with a much higher proportion of meso/macropores (Fig. S18-S20†). Surprisingly, the CV curve of GPC-1000-5 showed a significantly negatively shifted peak potential of $-0.25 \mathrm{~V}$, accompanied by the $E_{\text {onset }}$ at $-0.09 \mathrm{~V}$, indicating a much inferior ORR activity. However, GPC-1000-5 exhibited a much higher $J_{\mathrm{L}}\left(5.7 \mathrm{~mA} \mathrm{~cm}^{-2}\right)$, which outperforms that of commercial Pt/C. Moreover, it has a similar $J_{\mathrm{K}}$ value of $19.6 \mathrm{~mA} \mathrm{~cm} \mathrm{~cm}^{-2}$ to that of $\mathrm{Pt} / \mathrm{C}$ at $-0.35 \mathrm{~V}$, indicating its potentially high activity (see Fig. $5 \mathrm{f}$ ).

The results obtained clearly show that solely MOF-derived heteroatom-doped carbon materials can be applied as an efficient metal-free ORR catalyst in fuel cells. The necessity of a fundamental understanding of the structural characters of the NGPCs towards ORR enables us to have a brief discussion regarding their ORR performance and mesoscopic characters. The enhanced electrocatalytic activity is clearly witnessed from two important features: a significant positive shift of the oxygen reduction potential and an increase in the oxygen reduction current density, both of which are closely related to the ORR active sites. Of the four nitrogen species, pyridinic-N, pyrrolic-N and graphitic- $\mathrm{N}$ were widely reported to be the ORR active sites, respectively, albeit controversy still exists. ${ }^{51}$ However, among the NGPCs, NGPC-700-5 with the maximum amount of pyrrolic$\mathrm{N}$ exhibited the poorest ORR performance, while NGPC-1000-10 with a negligible amount of pyrrolic-N exhibited the highest ORR performance, indicating the pyrrolic-N may contribute little to the ORR activity. Thus, the pyridinic-N and graphitic-N are considered in tandem, and the ratios of $(\mathrm{N} 1+\mathrm{N} 3) / \mathrm{N}_{\text {total }}$ of different samples are listed in Table S1.† Surprisingly, when coupled with the ORR experimental data, we can observe that the ratio of $(\mathrm{N} 1+\mathrm{N} 3) / \mathrm{N}_{\text {total }}$ positively correlates to the $E_{\text {onset }}$ values of NGPCs obtained at different $T_{\mathrm{c}}$. This correlation cannot be considered as conclusive quantitative judgment due to the complexity of ORR catalysis. However, it does point to the substantial contributions of pyridinic- and graphitic-N species, presumably by promoting electron transfer and $\mathrm{O}_{2}$-adsorption in ORR catalysis. ${ }^{51,52}$ Notably, the NGPCs obtained at $1000{ }^{\circ} \mathrm{C}$ have similar $(\mathrm{N} 1+\mathrm{N} 3) / \mathrm{N}_{\text {total }}$ ratios $(0.87-0.90)$, but the $E_{\text {onset }}$ of NGPC-1000-10 is significantly higher than those of NGPC-10001 and NGPC-1000-5. This can be correlated to the increased portion of graphitic- $\mathrm{N}$ as the continuous transformation of pyridinic- $\mathrm{N}$ to graphitic- $\mathrm{N}$ at prolonged time, suggesting that graphitic-N may contribute more than pyridinic-N to the ORR

Table 2 Summary of the electrochemical properties of ORR catalysts

\begin{tabular}{|c|c|c|c|c|c|c|}
\hline Sample & $E_{\text {onset }}{ }^{a} / \mathrm{mV}$ & $E_{1 / 2}^{b} / \mathrm{mV}$ & $\left|J_{\mathrm{L}}\right|^{c} / \mathrm{mA} \mathrm{cm}^{-2}$ & $\left|J_{\mathrm{K}}\right|^{d} / \mathrm{mA} \mathrm{cm}^{-2}$ & $n^{e}$ & $\mathrm{H}_{2} \mathrm{O}_{\text {selectivity }} / \%$ \\
\hline NGPC-700-5 & $-212 /-540$ & -441 & 2.78 & 0.86 & 2.20 & 12.0 \\
\hline NGPC-800-5 & -158 & -409 & 4.15 & 1.65 & 3.29 & 65.0 \\
\hline NGPC-900-5 & -91 & -293 & 4.99 & 5.88 & 3.38 & 71.5 \\
\hline NGPC-1000-1 & -39 & -223 & 4.45 & 8.54 & 3.65 & 85.0 \\
\hline NGPC-1000-5 & -45 & -234 & 4.31 & 7.70 & 3.45 & 75.5 \\
\hline NGPC-1000-10 & -17 & -198 & 4.69 & 14.18 & 3.81 & 91.0 \\
\hline GPC-1000-5 & -89 & -260 & 5.66 & 19.60 & 3.02 & 52.5 \\
\hline $20 \mathrm{wt} \% \mathrm{Pt} / \mathrm{C}$ & 20 & -164 & 4.58 & 19.87 & 3.92 & 96.5 \\
\hline
\end{tabular}

${ }^{a}$ Onset potential. ${ }^{b}$ Half-wave potential. ${ }^{c}$ Limiting current density, obtained at $-1.0 \mathrm{~V}$ with the rotation speed of $1600 \mathrm{rpm}$, otherwise the Pt/C at $-0.80 \mathrm{~V} .{ }^{d}$ Kinetic current density calculated at $-0.35 \mathrm{~V} .{ }^{e}$ Electron transfer number calculated at $-0.35 \mathrm{~V}$ based on the K-L equation. ${ }^{f}$ Determined by using the currents measured from both the ring and disk at $-0.35 \mathrm{~V}, 1600 \mathrm{rpm}$. 
activity. The more active character of graphitic- $\mathrm{N}$ species is verified by the larger $n$ values of NGPCs obtained at higher temperatures. Kim et al. proposed that the graphitic-N species located at the zigzag edge of graphene should be the main active site for the enhanced first electron transfer rate and the preference of the four-electron reduction pathway through a theoretic calculation method. ${ }^{52}$ In yet another report, Luo et al. synthesized purely pyridinic-N doped graphenes and found them to be selective for a two-electron reduction pathway. ${ }^{53}$ The average $n$ values of 2.12 and 3.84 for NGPC-700-5 with predominant pyridinic-N and NGPC-1000-10 with the highest amount of graphitic-N, respectively, well coincides with those reports where graphitic- $\mathrm{N}$ plays a more important role in the enhanced ORR activity in alkaline solution than pyridinic-N. ${ }^{\mathbf{5 4 , 5 5}}$ However, the nitrogen species seem to be not the only active site that determines the $E_{\text {onset }}$ values of NGPCs, since both the slightly higher $(\mathrm{N} 1+\mathrm{N} 3) / \mathrm{N}_{\text {total }}$ ratio and graphitic- $\mathrm{N}$ portion in NGPC-800-5 resulted in much enhanced $E_{\text {onset }}$ and average $n$ value compared with those of NGPC-700-5. Meanwhile, the nitrogen-free GPC-1000-5 exhibited a competitive $E_{\text {onset }}$ of -0.09 $\mathrm{V}$ and average $n$ value of 3.12 , which is significantly higher than those reported for nitrogen-free CNTs whose ORR usually proceeds in an obvious two-step two-electron pathway with a much negative shift of $E_{\text {onset }}{ }^{56,57}$ Britto et al. found that the defects on CNTs surface were responsible for the two-electron oxygen reduction through the combined results of experimental and theoretical studies. ${ }^{58}$ In a further study of eight different carbon materials by $\mathrm{Xu}$ et al., it was found that both the defects and reduced oxygen-containing groups on the carbon surface were the electroactive sites, which can electrocatalyze the reduction of the $\mathrm{H}_{2} \mathrm{O}_{2}$ intermediates produced in the reduction of molecular $\mathrm{O}_{2}$ by Pt. ${ }^{59}$ Furthermore, Silva et al. synthesized Nand O-doped carbons derived from polyaniline with an unprecedented high ORR activity which was attributed to the synergistic effect of the $\mathrm{N}$ and $\mathrm{O}$ doping. ${ }^{\mathbf{6 0}}$ Therefore, the higher $E_{\text {onset }}$ and average $n$ value of NGPC-800-5 than those of NGPC700-5 can be reasonably ascribed to the structural defects formed during the carbonization process at $800{ }^{\circ} \mathrm{C}$, at which temperature the zinc species were reduced followed by the rearrangement of carbon atoms to gradually form a nitrogendoped graphitic carbon framework, ${ }^{28}$ hence causing a large number of structural defects on the carbon surface, which was explicitly revealed by the highest $I_{\mathrm{D}} / I_{\mathrm{G}}$ ratio of 1.26 in the Raman spectrum (Fig. S3†). Besides, the less effective nitrogen doping in the less graphitic carbon frameworks may also play an important role in the inferior ORR performance of NGPC-700-5. While for GPC-1000-5, its electroactive sites may be tentatively attributed to the oxygen-containing species on the surface or the doped oxygen atoms bonded to carbon or both, since it has a high amount of oxygen species of $23.82 \mathrm{wt} \%$ calculated from elemental analysis (wt\%, C 75.16; $\mathrm{H} 1.02)$. The active sites of ORR on GPC-1000-5 need to be further investigated in our future paper.

Furthermore, with respect to the current density, it is found that with $T_{\mathrm{c}}$ increasing from 700 to $1000{ }^{\circ} \mathrm{C}$, the corresponding $J_{\mathrm{L}}$ is closely related to the SSA of NGPC, namely, NGPC-900-5 with the highest SSA generally exhibits the highest $J_{\mathrm{L}}$ due to the large amount of exposed active sites and fast mass transportation. Notably, the SSA of NGPC-1000-5 is 1.2 times that of NGPC-800-5. However, the corresponding $J_{\mathrm{L}}$ of the latter is slightly lower than that of former. This is largely due to the higher nitrogen doping level resulting in more active sites exposed in the NGPC-800-5 than in NGPC-1000-5. For the NGPCs obtained at $1000{ }^{\circ} \mathrm{C}$ with different carbonization times, their SSA values gradually decreased in a small range of 40-50 $\mathrm{m}^{2} \mathrm{~g}^{-1}$ from 1 to $10 \mathrm{~h}$, but their $J_{\mathrm{L}}$ values were in the order NGPC-1000-5 < NGPC-1000-1 < NGPC-1000-10. As is well known, the conductivity of materials can greatly influence the electron transfer rate, thus, a high degree of graphitization (good electrical conductivity) is a favorable factor for ORR performance of NGPCs with close nitrogen contents (active sites) and SSA values, which leads to the superior ORR performance for NGPC1000-10 than that of NGPC-1000-5; while the lower $J_{\mathrm{L}}$ coupled with higher degree of graphitization of NGPC-1000-5 compared with those of NGPC-1000-1 can be rationally ascribed to the lower amount of active sites and the loss of SSA during the long duration of pyrolysis. This can also be used to explain the highest $J_{\mathrm{L}}$ of GPC-1000-5, which has both the highest degree of graphitization and SSA.

The superior $J_{\mathrm{L}}$ coupled with the inferior $E_{\text {onset }}$ of GPC-1000-5 compared with that of NGPC-1000-5 signifies that the introduction of nitrogen atoms into MOF-derived carbons greatly enhances the intrinsic oxygen reduction properties of these materials with the reduction of overpotential, thus leading to excellent ORR activity that approximates the performance of commercial Pt/C. Along with previous reports, we speculate that the superior electrocatalytic activity of NGPC-1000-5 compared with that of nitrogen-free GPC-1000-5 can be attributed to the lower $\mathrm{O}_{2}$ adsorption and activation energy resulting from nitrogen doping. ${ }^{61,62}$ However, it is not a case of the more nitrogen doping the better. As displayed in Fig. 5e, NGPC-700-5, NGPC-800-5 and NGPC-900-5 with higher nitrogen content than that of NGPC-1000-5, actually exhibited lower selectivity and catalytic activity, which indicates that higher activity is not proportional to the total amount of nitrogen, which is in agreement with previous reports. ${ }^{16,55}$ However, samples with a higher concentration of $\mathrm{N}$ synthesized in lower temperature have a lower SSA as well as conductivity, although these are crucial in electrocatalytic process. From the viewpoint of doping, $\mathrm{N}$ provides more valence electrons than $\mathrm{C}$, and their substitution for carbon up to a certain concentration increases the density of conducting electrons at energy near the Fermi level, thus promoting conductivity and catalytic activity. The $\mathrm{N}$ dopants are, however, more electronegative than carbon, and overdoping could result in localization of conducting electrons at the dopant site and finally lead to loss of conductivity. ${ }^{\mathbf{1 2}}$ Furthermore, in a theoretic study, it has been reported that model graphene with a higher nitrogen content was more easily poisoned by adsorbed $\mathrm{O}_{2}$ because of the stronger affinity. ${ }^{63}$ These results suggest an optimal nitrogen content might be critical to achieve a high ORR activity.

Now we could rationally understand the difference in catalytic ability for the various nitrogen-doped and nitrogen-free graphitic porous carbons demonstrated in the experimental 


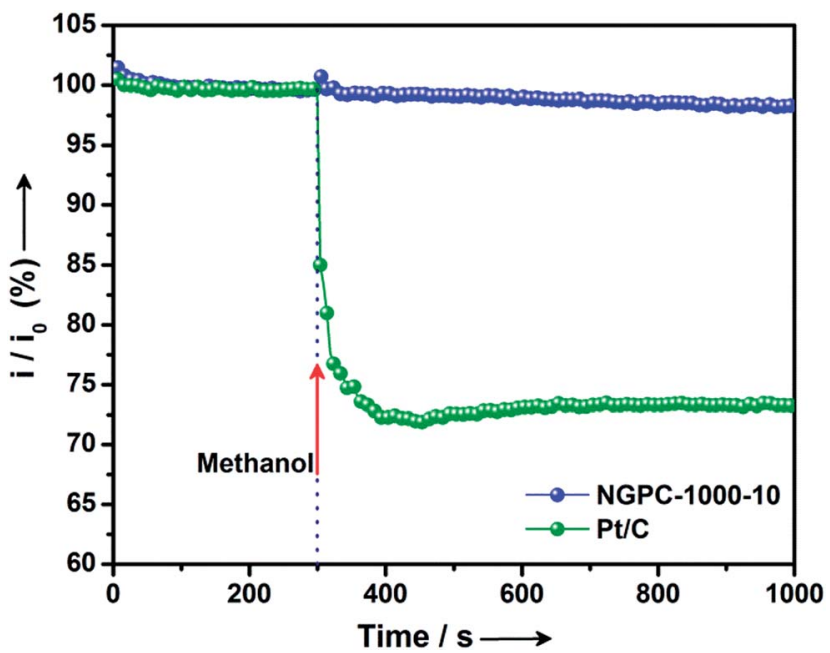

Fig. 6 Current-time $(i-t)$ chronoamperometric responses at $-0.4 \mathrm{~V}$ in $\mathrm{O}_{2}$-saturated $0.1 \mathrm{M} \mathrm{KOH}$ on NGPC-1000-10 and Pt/C electrodes (1600 rpm) followed by the introduction of methanol (3 M) at $300 \mathrm{~s}$.

measurements. Obviously it is the synergistic effect, which includes abundant catalytic sites for ORR provided by high $\mathrm{N}$ heteroatom loading, excellent reactant transport caused by hierarchical pore structures and high electron transfer rate provided by $3 \mathrm{D}$ continuous well-conducting networks, thus leading to the optimum performances for the NGPC-1000-10.

To further investigate the effect of zinc residue in the NGPCs on their catalysis for ORR, samples of NGPC-800-5 with and without acid leaching treatment were subjected to the ORR study. It is interesting that the purified NGPC-800-5 showed almost same activity for ORR (same $E_{\text {onset }}$ ), even slightly higher $J_{\mathrm{L}}$ (Fig. S21 $\dagger$ ), indicating that zinc residues may not contribute to the ORR or contribute only a little. Moreover, the enhanced $J_{\mathrm{L}}$ may result from the more active sites exposed caused by the removal of zinc impurities.

Along with the electrocatalytic activity for the ORR, the NGPC-1000-10 catalyst also presents excellent cycling stability and immunity towards methanol crossover, which overcomes another main challenge faced by metal-based catalysts in fuel cells. The corresponding $i-t$ chronoamperometric responses to methanol introduced into the $\mathrm{O}_{2}$-saturated electrolyte were analyzed for both NGPC-1000-10 and $20 \mathrm{wt} \% \mathrm{Pt} / \mathrm{C}$ catalysts (Fig. 6). After the addition of $3 \mathrm{M}$ methanol, the ORR current for the $\mathrm{Pt} / \mathrm{C}$ catalyst suffered a sharp decrease as a result of the mixed potential (Fig. S22 $\dagger$ ), suggesting that $\mathrm{Pt} / \mathrm{C}$ is active for methanol electrooxidation and is very sensitive to the fuel crossover effect. Remarkably, NGPC-1000-10 showed only a small attenuation in ORR current, suggesting an excellent selectivity for ORR to the evade crossover effect of the methanol and thereby outperforms even the commercial Pt/C electrode. This can be attributed to the effect of the hierarchically porous structure on the smooth diffusion rate of the electrolyte and the much lower ORR potential than that required for oxidation of the fuel molecules, which is further evidenced by the CV measurements in the presence and absence of $3 \mathrm{M}$ methanol (Fig. S23 $\dagger$ ). This methanol inertness matches well with
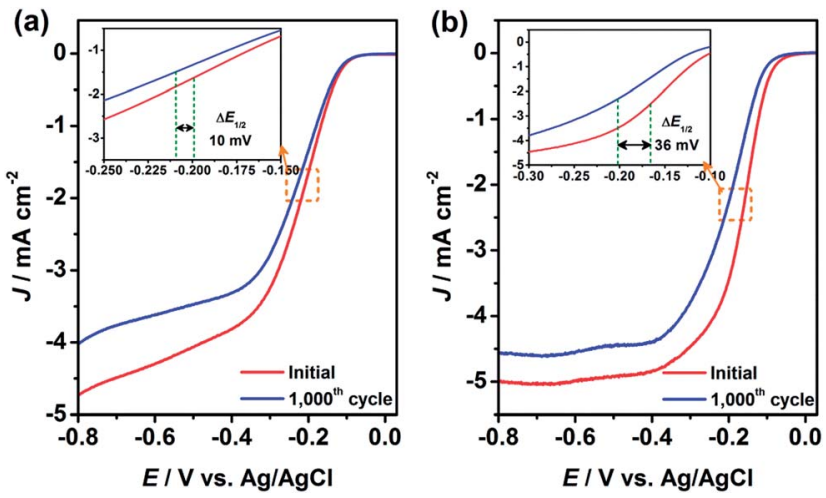

Fig. 7 Accelerated durability tests (ADTs) for (a) NGPC-1000-10 and (b) $20 \mathrm{wt} \% \mathrm{Pt} / \mathrm{C}$ with a rotation speed of $1600 \mathrm{rpm}$, and a sweep rate of $5 \mathrm{mV} \mathrm{s}^{-1}$

chemical expectations for the catalyst of such systems, suggesting that NGPCs-1000-10 is an ideal electrocatalyst for practical applications.

To evaluate the stability of the NGPC-1000-10, accelerated durability tests (ADTs) were performed by continuous potential cycling within a potential range of -0.8 to $0 \mathrm{~V}$ in $0.1 \mathrm{M} \mathrm{KOH}$, and with the $20 \mathrm{wt} \% \mathrm{Pt} / \mathrm{C}$ catalyst as a reference. Fig. 7 shows the polarization curves for ORR of the different catalysts before and after ADT. After 1000 cycles, the NGPC-1000-10 retained more than $80 \%$ of its initial current density at $-0.2 \mathrm{~V}$ with only $10 \mathrm{mV}$ negative shift in ORR activity in $E_{1 / 2}$. In contrast, the $\mathrm{Pt} / \mathrm{C}$ exhibited continuous current density fading upon prolonged cycling, with a current density loss of approximately $65 \%$ at -0.2 $\mathrm{V}$ and an $E_{1 / 2}$ drop of $36 \mathrm{mV}$. This indicates that the stability of NGPC-1000-10 is superior to that of the Pt/C catalyst. The higher stability of NGPC-1000-10 may be attributed to the nature of its active sites induced by nitrogen doping, because the strength of the covalent $\mathrm{C}-\mathrm{N}$ bond is well above the adsorption forces exerted between the noble- or non-noble-metal catalysts and their supports. ${ }^{64}$ Besides, the metal-free carbon catalyst does not suffer activity degradation caused by metal nanoparticle agglomeration and loss as Pt/C, which may contribute to their high stability. ${ }^{65}$ Moreover, the formation of graphitic carbon could also play a significant role in the improvement of the corrosion resistance for the carbon-based catalyst. ${ }^{66}$

\section{Conclusion}

In summary, we have demonstrated the fabrication of in situ nitrogen-doped carbon nanopolyhedra which feature high degree of graphitization, high surface area and hierarchical porosity by a facile low-cost and readily reproducible NMOFdriven approach. The resultant NGPCs serve as true metal-free catalysts that exhibit high electrocatalytic performance for ORR, coupled with enhanced stability and immunity towards fuel crossover than commercial Pt/C catalyst. Our study reveals that solely MOF without additional metal species or carbon sources can be used as an ideal precursor to afford efficient metal-free and heteroatom-doped carbon electrocatalysts for ORR in alkaline media. Generally, higher activity is expected for NGPC 
prepared at higher temperature, while longer carbonization time does not proportionally correlated to higher electroactivity. Moreover, the optimum electrocatalytic activity of NGPC-100010 among other NGPCs can be attributed to the synergistic contributions of its high SSA, high degree of graphitization, and high proportion of graphitic nitrogen atoms. The present findings are a striking indication towards the highly desirable carbon-based metal-free electrocatalysts for ORR. With the great number of available and rapidly growing MOF structures, MOFbased heteroatom-doped porous carbon materials with tailorable pore textures and improved performances could be highly promising. In-depth studies of MOF-derived carbon-based ORR electrocatalysts are in progress in our group.

\section{Acknowledgements}

The authors gratefully acknowledge the financial support of the 973 Program of China (2011CBA00507), the NSFC (Project nos 21131006 and 21371169), and the Natural Science Foundation of Fujian Province (2012H0047).

\section{Notes and references}

1 Y. Wang, K. S. Chen, J. Mishler, S. C. Cho and X. C. Adroher, Appl. Energy, 2011, 88, 981-1007.

2 B. C. Steele and A. Heinzel, Nature, 2001, 414, 345-352.

3 X. Yu and S. Ye, J. Power Sources, 2007, 172, 145-154.

4 Z. Chen, D. Higgins, A. Yu, L. Zhang and J. Zhang, Energy Environ. Sci., 2011, 4, 3167-3192.

5 A. Morozan, B. Jousselme and S. Palacin, Energy Environ. Sci., 2011, 4, 1238-1254.

6 N. Zhang, Y. Zhang and Y. J. Xu, Nanoscale, 2012, 4, 57925813.

7 D. Yu, E. Nagelli, F. Du and L. Dai, J. Phys. Chem. Lett., 2010, 1, 2165-2173.

8 A. Morozan, P. Jégou, M. Pinault, S. Campidelli, B. Jousselme and S. Palacin, ChemSusChem, 2012, 5, 647-651.

9 Y. Zhao, L. Yang, S. Chen, X. Wang, Y. Ma, Q. Wu, Y. Jiang, W. Qian and Z. Hu, J. Am. Chem. Soc., 2013, 135, 1201-1204.

10 D. Yu, Y. Xue and L. Dai, J. Phys. Chem. Lett., 2012, 3, 28632870 .

11 Z. W. Liu, F. Peng, H. J. Wang, H. Yu, W. X. Zheng and J. Yang, Angew. Chem., Int. Ed., 2011, 50, 3257-3261.

12 J. Liang, Y. Jiao, M. Jaroniec and S. Z. Qiao, Angew. Chem., Int. Ed., 2012, 51, 11496-11500.

13 I. Y. Jeon, S. Zhang, L. Zhang, H. J. Choi, J. M. Seo, Z. Xia, L. Dai and J. B. Baek, Adv. Mater., 2013, 25, 6138-6145.

14 J. Xu, G. Dong, C. Jin, M. Huang and L. Guan, ChemSusChem, 2013, 6, 493-499.

15 T. Hibino, K. Kobayashi and P. Heo, Electrochim. Acta, 2013, 112, 82-89.

16 R. Liu, D. Wu, X. Feng and K. Müllen, Angew. Chem., Int. Ed., 2010, 49, 2565-2569.

17 X. Sun, Y. Zhang, P. Song, J. Pan, L. Zhuang, W. Xu and W. Xing, ACS Catal., 2013, 3, 1726-1729.

18 J. Liang, X. Du, C. Gibson, X. W. Du and S. Z. Qiao, Adv. Mater., 2013, 25, 6226-6231.
19 P. Chen, T. Y. Xiao, Y. H. Qian, S. S. Li and S. H. Yu, Adv. Mater., 2013, 25, 3192-3196.

20 Y. Shao, J. Sui, G. Yin and Y. Gao, Appl. Catal., B, 2008, 79, 89-99.

21 H. Wang, T. Maiyalagan and X. Wang, ACS Catal., 2012, 2, 781-794.

22 H. A. Gasteiger, S. S. Kocha, B. Sompalli and F. T. Wagner, Appl. Catal., B, 2005, 56, 9-35.

23 S. T. Meek, J. A. Greathouse and M. D. Allendorf, Adv. Mater., 2011, 23, 249-267.

24 B. Liu, H. Shioyama, T. Akita and Q. Xu, J. Am. Chem. Soc., 2008, 130, 5390-5391.

25 A. Almasoudi and R. Mokaya, J. Mater. Chem., 2012, 22, 146152.

26 H. L. Jiang, B. Liu, Y. Q. Lan, K. Kuratani, T. Akita, H. Shioyama, F. Zong and Q. Xu, J. Am. Chem. Soc., 2011, 133, 11854-11857.

27 P. Pachfule, B. P. Biswal and R. Banerjee, Chem.-Eur. J., 2012, 18, 11399-11408.

28 B. Liu, H. Shioyama, H. Jiang, X. Zhang and Q. Xu, Carbon, 2010, 48, 456-463.

29 J. Hu, H. Wang, Q. Gao and H. Guo, Carbon, 2010, 48, 35993606.

30 G. Xu, B. Ding, L. Shen, P. Nie, J. Han and X. Zhang, J. Mater. Chem. A, 2013, 1, 4490-4496.

31 K. Xi, S. Cao, X. Peng, C. Ducati, R. Vasant Kumar and A. K. Cheetham, Chem. Commun., 2013, 49, 21922194.

32 P. Su, H. Xiao, J. Zhao, Y. Yao, Z. Shao, C. Li and Q. Yang, Chem. Sci., 2013, 4, 2941-2946.

33 S. J. Yang, T. Kim, J. H. Im, Y. S. Kim, K. Lee, H. Jung and C. R. Park, Chem. Mater., 2012, 24, 464-470.

34 M. Hu, J. Reboul, S. Furukawa, N. L. Torad, Q. Ji, P. Srinivasu, K. Ariga, S. Kitagawa and Y. Yamauchi, J. Am. Chem. Soc., 2012, 134, 2864-2867.

35 A. J. Amali, J. K. Sun and Q. Xu, Chem. Commun., 2014, 50, 1519-1522.

36 S. Ma, G. A. Goenaga, A. V. Call and D.-J. Liu, Chem.-Eur. J., 2011, 17, 2063-2067.

37 T. Palaniselvam, B. P. Biswal, R. Banerjee and S. Kurungot, Chem.-Eur. J., 2013, 19, 9335-9342.

38 K. S. Park, Z. Ni, A. P. Côté, J. Y. Choi, R. Huang, F. J. UribeRomo, H. K. Chae, M. O'Keeffe and O. M. Yaghi, Proc. Natl. Acad. Sci. U. S. A., 2006, 103, 10186-10191.

39 K. Stańczyk, R. Dziembaj, Z. Piwowarska and S. Witkowski, Carbon, 1995, 33, 1383-1392.

40 D. Banham, F. Feng, K. Pei, S. Ye and V. Birss, J. Mater. Chem. A, 2013, 1, 2812-2820.

41 J. Cravillon, R. Nayuk, S. Springer, A. Feldhoff, K. Huber and M. Wiebcke, Chem. Mater., 2011, 23, 2130-2141.

42 H. Li, M. Eddaoudi, M. O'Keeffe and O. M. Yaghi, Nature, 1999, 402, 276-279.

43 A. Sadezky, H. Muckenhuber, H. Grothe, R. Niessner and U. Pöschl, Carbon, 2005, 43, 1731-1742.

44 J. Cravillon, R. Nayuk, S. Springer, A. Feldhoff, K. Huber and M. Wiebcke, Chem. Mater., 2011, 23, 2130-2141.

45 A. Ōya and H. Marsh, J. Mater. Sci., 1982, 17, 309-322. 
46 Q. Wang, W. Xia, W. Guo, L. An, D. Xia and R. Zou, Chem.Asian J., 2013, 8, 1879-1885.

47 X. Liu, L. Zhou, Y. Zhao, L. Bian, X. Feng and Q. Pu, ACS Appl. Mater. Interfaces, 2013, 5, 10280-10287.

48 Z. Jiang, H. Sun, Z. Qin, X. Jiao and D. Chen, Chem. Commun., 2012, 48, 3620-3622.

49 Z. Chen, D. Higgins and Z. Chen, Carbon, 2010, 48, 30573065.

50 T. C. Nagaiah, A. Bordoloi, M. D. Sánchez, M. Muhler and W. Schuhmann, ChemSusChem, 2012, 5, 637-641.

51 W. Ding, Z. Wei, S. Chen, X. Qi, T. Yang, J. Hu, D. Wang, L. J. Wan, S. F. Alvi and L. Li, Angew. Chem., Int. Ed., 2013, 52, 11755-11759.

52 H. Kim, K. Lee, S. I. Woo and Y. Jung, Phys. Chem. Chem. Phys., 2011, 13, 17505-17510.

53 Z. Luo, S. Lim, Z. Tian, J. Shang, L. Lai, B. MacDonald, C. Fu, Z. Shen, T. Yu and J. Lin, J. Mater. Chem., 2011, 21, 8038-8044.

54 H. Niwa, K. Horiba, Y. Harada, M. Oshima, T. Ikeda, K. Terakura, J.-i. Ozaki and S. Miyata, J. Power Sources, 2009, 187, 93-97.

55 T. C. Nagaiah, S. Kundu, M. Bron, M. Muhler and W. Schuhmann, Electrochem. Commun., 2010, 12, 338-341.
56 L. Yang, S. Jiang, Y. Zhao, L. Zhu, S. Chen, X. Wang, Q. Wu, J. Ma, Y. Ma and Z. Hu, Angew. Chem., Int. Ed., 2011, 50, 7132-7135.

57 K. Gong, F. Du, Z. Xia, M. Durstock and L. Dai, Science, 2009, 323, 760-764.

58 P. J. Britto, K. S. Santhanam, A. Rubio, J. A. Alonso and P. M. Ajayan, Adv. Mater., 1999, 11, 154-157.

59 W. Xu, X. Zhou, C. Liu, W. Xing and T. Lu, Electrochem. Commun., 2007, 9, 1002-1006.

60 R. Silva, D. Voiry, M. Chhowalla and T. Asefa, J. Am. Chem. Soc., 2013, 135, 7823-7826.

61 B. Shan and K. Cho, Chem. Phys. Lett., 2010, 492, 131136.

62 X. Hu, Y. Wu, H. Li and Z. Zhang, J. Phys. Chem. C, 2010, 114, 9603-9607.

63 Y. Okamoto, Appl. Surf. Sci., 2009, 256, 335-341.

64 Z. Lin, G. Waller, Y. Liu, M. Liu and C. P. Wong, Adv. Energy Mater., 2012, 2, 884-888.

65 Y. Shao-Horn, W. Sheng, S. Chen, P. Ferreira, E. Holby and D. Morgan, Top. Catal., 2007, 46, 285-305.

66 J. Zeng, F. Su, J. Y. Lee, W. Zhou and X. Zhao, Carbon, 2006, 44, 1713-1717. 